\title{
Epigenetic regulation and reprogramming: from embryos, to cloned embryos and to embryonic stem cells
}

\author{
Fanyi Zeng $^{1}$

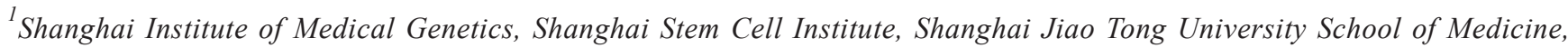 \\ Shanghai, China
}

Preimplantation embryo development is marked with pronounced reprogramming of gene expression patterns during distinct transitions, namely the maternal-to-zygotic transition, compaction and blastocyst formation. Using microarray analysis, combined with functional genomics tools, we characterized global changes in gene expression and identified biological processes in mouse early embryo stages that accompany and likely underlie these transitions. For example, fertilization results in changes in the transcript profile in the 1-cell embryo that are far greater than previously recognized, and genome activation during 2-cell stage are quite selective rather than global and promiscuous, with genes involved in transcription and RNA processing being preferentially expressed. Nuclear transfer (NT) provides a useful model to further study the important reprogramming events in the early embryo, especially the maternal to zygote interrelationship. Since mitochondria $(\mathrm{mt})$ are the most abundant maternallyinherited organelle, the effect of mtDNA haplotypes on bovine NT efficiency was evaluated. Our data suggests that mitochondrial structure, quantity and function may significantly affect the developmental competence of reconstructed embryos. As embryonic stem cells (ESCs) from cloned embryos would provide powerful research and clinical tools, we derived forty NT-ESC lines from NT embryos of different donor cell types or passages. These NTESCs expressed pluripotency stem cell markers in vitro and could differentiate into various embryonic tissues in vitro and in vivo. These studies of preimplantation embryo gene expression, epigenetic regulation, and their impact on ESC derivation provide a framework for understanding early development and stem cell biology.

Keywords: preimplantation embryo, expression profiling, nuclear transfer, mitochondria, embryonic stem cell

Cell Research (2008) 18:s21. doi: 10.1038/cr.2008.111; published online 4 August 2008

Correspondence: Fanyi Zeng

E-mail: f.zeng@verizon.net

Fanyi Zeng, MD/PhD, received her MD and PhD degree from University of Pennsylvania. She is current Professor and $\mathrm{PhD}$ advisor at Shanghai Institute of Medical Genetics, Shanghai Children's Hospital, Deputy Director of Shanghai Institute of Medical Genetics, and Associate
Director of Shanghai Stem Cell Institute, Shanghai Jiao Tong University, China. Dr Zeng is also the Chief Scientist of National Basic Research Program of China, and Principal Investigator of National Natural Science Foundation of China, Shanghai Pu-Jiang Scholar Plan and Shanghai ShuGuang Plan. Her research focuses on the differentiation of human stem cells, gene expression profiling of preimplantation embryos as well as somatic cell cloning. 